\title{
Tattoo-associated Mycobacterium franklinii infection
}

\author{
Campbell L Stewart ${ }^{1}$, Danielle M DeHoratius ${ }^{3}$, Stephen Gluckman ${ }^{4}$, Christine Rongey ${ }^{5}$, Kevin Alby ${ }^{6}$, \\ Rosalie Elenitsas ${ }^{2 *}$ \\ ${ }^{1}$ Department of Medicine, Division of Dermatology, University of Washington, Seattle, WA \\ ${ }^{2}$ Department of Dermatology, University of Pennsylvania, Philadelphia, PA \\ ${ }^{3}$ Dermatology Associates of Bryn Mawr, Bryn Mawr, PA \\ ${ }^{4}$ Department of Medicine, Division of Infectious Diseases, University of Pennsylvania, Philadelphia, PA \\ ${ }^{5}$ Department of Pathology, UCLA Medical Center, Los Angeles, CA \\ ${ }^{6}$ Department of Pathology, University of Pennsylvania, Philadelphia, PA
}

Received: November 24, 2016; Accepted: December 06, 2016; Published: January 06, 2017

*Corresponding author: Dr. Rosalie Elenitsas, Department of Dermatology, University of Pennsylvania, Philadelphia, PA. E-mail: rosalie. elenitsas@uphs.upenn.edu

\begin{abstract}
Importance: Tattoo-associated cutaneous mycobacterial infections have been reported with increasing frequency. The majority of these have been associated with $M$. chelonae, a rapidly growing nontuberculous mycobacterium. M. franklinii is a newly described member of the $M$. chelonae-abscessus group, which can cause upper respiratory and rare skin infections. It has a distinct genetic profile and anti-microbial sensitivities. Unlike $M$. chelonae, $M$ franklinii may carry the erm gene, which confers inducible macrolide antibiotic resistance. Therefore, localized infection cannot be treated with macrolide monotherapy.
\end{abstract}

Observations: We report a case of a 36 year-old African American man who developed an eruption of asymptomatic papules at the site of a tattoo he received 1 month previously. A punch biopsy demonstrated mixed inflammation with a small focus of acid-fast positive organisms. Tissue culture and genetic testing using rpoB sequencing identified the organism as $M$. franklinii. Based on these results, he was successfully treated with a combination of doxycycline and clarithromycin.

Conclusions and Relevance: It is important for dermatologists to recognize newly described non-tuberculous mycobacterial causes of cutaneous infection, and consider sending specimens for genetic testing in addition to tissue culture. Precise identification of these mycobacteria can lead to appropriate anti-microbial coverage and improved response to treatment.

Keywords: Mycobacterium franklenii; Tattoo; Infection; Nontuberculous Mycobacterium; Dermatopathology;

\section{Report of a case}

A 36 year-old African American man with no remarkable medical history had an amateur tattoo performed on his left upper arm. Within 1 month, he developed small asymptomatic skin colored papules clustered in and around the tattoo (Figure 1). A trial of $1 \%$ hydrocortisone ointment was ineffective and new papules continued to appear.

The patient was referred to dermatology 2 months later and a punch biopsy of his eruption was performed. The biopsy revealed a dense mixed inflammatory infiltrate in the dermis comprised of lymphocytes, histiocytes, plasma cells, and neutrophils (Figure 2A). Adjacent to the inflammatory infiltrate were large, coarse, black granules, consistent with tattoo pigment (Figure 2B). Acid-fast bacilli and Fite stains demonstrated a small cluster of mycobacterial organisms within the infiltrate (Figure 2C).

Based on the histopathology result, tissue was obtained for routine, mycobacterial, and fungal culture and the patient was started on oral ciprofloxacin and clarithromycin. Seven days later, the mycobacterial culture was positive for growth of an acid fast organism, subsequently identified as Mycobacterium franklinii, by $r p o B$ gene sequencing. The organism was susceptible to amikacin, doxycycline, minocycline, and clarithromycin. While the patient had not developed new lesions during the time between biopsy and culture result, the papules had persisted on oral antibiotic therapy. Based on the speciation result and his lack of improvement, he was referred to an infectious diseases specialist for further care and his regimen was altered to doxycycline and clarithromycin.

\section{Discussion}

M. franklinii is an acid-fast, gram positive bacillus. It is a recently described member of rapidly growing mycobacteria which cause skin and soft tissue infections, pneumonia, and bloodstream infections in both immune competent and immune

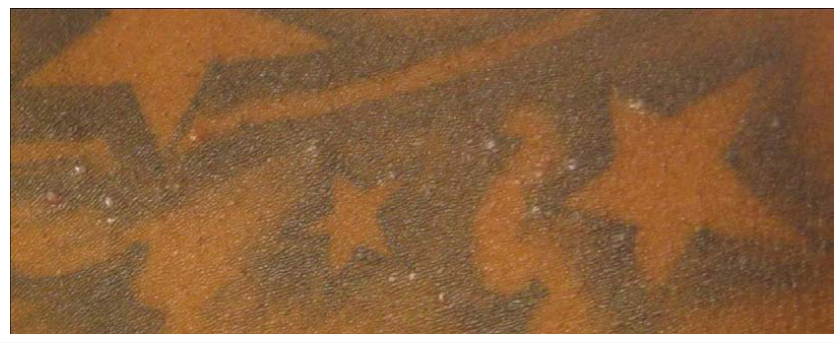

Figure 1: $M$. franklinii infection. Scattered skin colored papules clustered in and around a tattoo, concentrated in areas of gray shading. 
compromised hosts. Other rapidly growing mycobacteria include M. chelonae, M. immunogenum, and M. abscessus [1].

M. franklinii was first identified in 2007 as an atypical variant of M. chelonae, with clinical behavior similar to M. abscessus. It differed from $M$. chelonae in its susceptibility or intermediate resistance to cefoxitin. The morphology is similar to other rapidly growing mycobacteria, including both $M$. abscessus and M. chelonae. Additionally, M. franklinii cannot be readily distinguished from $M$. abscessus or M. chelonae by partial $16 \mathrm{~S}$ rDNA sequencing, and alternative targets such as $r p o B$, hsp65, or $\operatorname{sod} A$ must be used. The exact source of this pathogen has not been identified, however, municipal water sources have been implicated [1, 2]. Most isolates were found in Pennsylvania, and after testing confirmed the bacterium to be distinct from $M$. chelonae, the bacterium was named for Benjamin Franklin [1]. In 2011, Simmon et al. published a series of 26 patients, confirming M. franklinii as a novel species causing infection based on DNADNA hybridization, multilocus DNA target sequencing, and deep multilocus sequencing. Most infections occurred in patients with chronic lung disease, intravascular catheters, or chronic sinusitis. The majority of these patients had respiratory infections, however, two patients did have isolated skin infections [1].

Recently, rapidly growing mycobacterial skin infections associated with tattoos have been reported with increasing frequency $[3,4]$. These infections are most commonly attributed to the use of non-sterile water sources for the dilution of tattoo inks $[5,8]$. However, contamination can occur at any time from manufacture of the tattoo ink or equipment, to application, to post

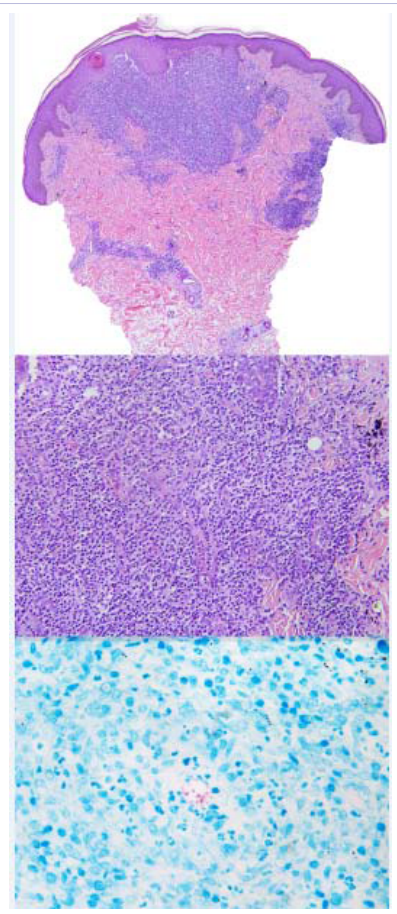

Figure 2: A: M. franklinii infection. Punch biopsy of a papule revealed dense dermal inflammation (40x). B: Mixed dermal inflammation with tattoo pigment at the periphery (200x). C: Fite stain highlights a small focus of mycobacteria (600x). tattoo wound care [5, 8]. A number of investigations identified rapidly growing mycobacteria or other bacteria in opened bottles of tattoo ink. In such cases, it is impossible to verify at what point the contamination has occurred. Only one of these reports was able to confirm the mycobacterial contamination in unopened bottles of tattoo ink, suggesting contamination during manufacture or distribution. This investigation lead to a manufacturer-based recall of these inks [7].

Based on a systematic review of tattoo associated rapid grower mycobacterial infections, $M$. chelonae is identified in nearly $70 \%$ of the cases [5]. Other commonly identified agents include M. Immogenum, M. abscessus, M. hemophilum, and $M$. fortuitum [8-10]. Our patient had an amateur tattoo performed in his friend's basement. He denied that any tap water had been used in his tattoo, however a pre-diluted gray ink was used. His infection developed within 1 month of exposure.

Over $20 \%$ of the adult population in the United States has at least one tattoo, and this number is increasing in young adults [6]. Dermatologists are likely to encounter increasing numbers of tattoo associated morbidities such as foreign body reactions, allergic hypersensitivity reactions, as well as infections. Therefore it is important for dermatologists to understand that tattoo associated mycobacterial infections can be caused by several subtypes with varied antibiotic sensitivity profiles. If a tattoo-associated mycobacterial infection is suspected and culture and histology are unrevealing, then repeated biopsy, culture and increased culture incubation times may be useful [7].

In conclusion, we present a rare case of tattoo associated M. franklinii infection. While there are not enough documented cases of $M$. franklinii skin infection to make treatment recommendations, doxycycline or minocycline in combination with clartithromycin are most likely to be effective. It is important to note that M. franklinii may carry an inducible erm gene, which may confer inducible clarithormycin resistance [1]. Therefore antibiotic monotherapy is not recommended, and referral to an infectious disease physician should be considered. The duration of treatment for M. franklinii is unstudied. Extrapolating from the management of other cutaneous mycobacterial infections treatment should continue for at least 1 month after resolution of skin findings.

\section{References}

1. Simmon KE, Brown-Elliott BA, Ridge PG, Durtschi JD, Mann LB, Slechta ES, et al. Mycobacterium chelonae-abscessus complex associated with sinopulmonary disease, Northeastern USA. Emerg Infect Dis. 2011;17(9):1692-1700. doi: 10.3201/eid1709.101667.

2. Van Ingen J, Blaak H, de Beer J, de Roda Husman AM, van Soolingen D. Rapidly growing nontuberculous mycobacteria cultured from home tap and shower water. Appl Environ Microbiol. 2010;76(17):60176019. doi: 10.1128/AEM.00843-10

3. Frew JW, Nguyen RT. Tattoo-associated mycobacterial infections: an emerging public health issue. Med J Aust. 2015;203(5):223-223e 221.

4. Simunovic C, Shinohara MM. Complications of decorative tattoos: recognition and management. Am J Clin Dermatol. 2014;15(6):525536. doi: 10.1007/s40257-014-0100-x. 
5. Centers for Disease C, Prevention. Tattoo-associated nontuberculous mycobacterial skin infections--multiple states, 2011-2012. MMWR Morb Mortal Wkly Rep. 2012;61(33):653-656.

6. Conaglen PD, Laurenson IF, Sergeant A, Thorn SN, Rayner A, Stevenson J. Systematic review of tattoo-associated skin infection with rapidly growing mycobacteria and public health investigation of a cluster in Scotland, 2010. Euro Surveill. 2013;18(32):20553.

7. Kennedy BS, Bedard B, Younge M, Deborah Tuttle, Eric Ammerman John Ricci, et al. Outbreak of Mycobacterium chelonae infection associated with tattoo ink. N Engl J Med. 2012;367(11):1020-1024. DOI: 10.1056/NEJMoa1205114
8. Muramatsu $\mathrm{K}$, Nomura T, Ito T, Hamade $Y$, Hirata $Y$, Fujita $Y$, et al Repeated skin sampling and prolonged incubation period identified cutaneous Mycobacterium chelonae infection on the face in an immunocompetent man. Br J Dermatol. 2014;170(2):471-473. doi: $10.1111 /$ bjd.12638

9. Mudedla S, Avendano EE, Raman G. Non-tuberculous mycobacterium skin infections after tattooing in healthy individuals: A systematic review of case reports. Dermatol Online J. 2015;21(6).

10. Philips RC, Hunter-Ellul LA, Martin JE, Wilkerson MG. Mycobacterium fortuitum infection arising in a new tattoo. Dermatol Online J. $2014 ; 20(6)$ 